\title{
Laparoscopic surgery to treat leiomyosarcomas of the sigmoid colon: a case report and literature review
}

\author{
Masashi Yahagi $i^{1}$, Yoshiyuki Ishii ${ }^{1,3^{*}}$, Atsuko Hara ${ }^{2}$ and Masahiko Watanabe ${ }^{1,3}$
}

\begin{abstract}
Background: Leiomyosarcomas (LMSs) of the colon are extremely rare and highly aggressive. Although treatment of gastrointestinal LMS is not standardized, surgical resection is generally performed. The fact that the tumors are usually large at the time of diagnosis may explain why no report on laparoscopic resection of a colonic LMS has appeared.

Case presentation: A 46-year-old male presented with hematochezia 1 month in duration. Abdominal examination including palpation was normal. The levels of several blood tumor markers were normal. Colonoscopy revealed a polypoid lesion approximately $30 \mathrm{~mm}$ in diameter in the sigmoid colon $30 \mathrm{~cm}$ from the anal verge. Contrast-enhanced computed tomography revealed that the tumor was $28 \mathrm{~mm}$ in diameter, and that no lymph node or distant metastasis was apparent. Histopathological examination of a biopsy specimen revealed spindle-shaped cells exhibiting significant nuclear atypia and a trabecular proliferation pattern upon hematoxylin-eosin staining. Immunohistochemically, the sample was positive for SMA and desmin, and negative for c-kit, DOG-1, CD34, and S-100. Furthermore, the Ki-67 index was $>50 \%$. We thus diagnosed a leiomyosarcoma of the sigmoid colon without any metastasis. We performed laparoscopic sigmoid colectomy and regional lymphadenectomy using five trocars. After complete curative resection, a colorectal end-to-end anastomosis was created employing the double-stapling technique. All surgical margins were negative, and no lymph node metastasis was observed. The postoperative course was uneventful, and the patient was discharged 9 days after operation. No recurrence was noted to 1 year after surgery.

Conclusions: We report the first case of a colonic LMS treated via laparoscopic surgery. Although further work is necessary to assess prognosis and to develop the treatment further, laparoscopic surgery to treat small colonic LMSs may be feasible, being both minimally invasive and curative.
\end{abstract}

Keywords: Leiomyosarcoma, Colon, Laparoscopic surgery

\section{Background}

Leiomyosarcoma (LMS) of the colon is an extremely rare and highly aggressive neoplasm $[1,2]$. After it was found that c-kit gain-of-function played an important oncological role in gastrointestinal stromal tumors (GISTs), many tumors previously diagnosed as LMSs turned out to be GISTs [3]. Today, true LMS is immunohistochemically distinguished from other mesenchymal tumors by virtue of the expression of smooth muscle actin (SMA) and

\footnotetext{
* Correspondence: yoshi6671@me.com

'Department of Surgery, Kitasato University Kitasato Institute Hospital, 5-9-1

Shirokane, Minato-ku, Tokyo, Japan

${ }^{3}$ Department of Surgery, Kitasato University School of Medicine, 1-15-1

Kitasato, Sagamihara, Kanagawa, Japan

Full list of author information is available at the end of the article
}

desmin, but not GIST markers (KIT, CD34, and DOG1) or the schwannoma marker (S100) [4]. As an LMS of the colon is less symptomatic than an LMS of the rectum, colonic tumors are often large [2], and no instance of laparoscopic resection has yet been reported. Here, we present the first case of LMS of the sigmoid colon that was safely and curatively resected via laparoscopic surgery.

\section{Case presentation}

A 46-year-old male presented to our institution complaining of hematochezia 1 month in duration. His past medical history included coronary vasospastic angina and diabetes mellitus that was treated with insulin. $\mathrm{He}$ drank socially and had a 50-pack-year smoking history, but had quit smoking 4 months prior. Abdominal 

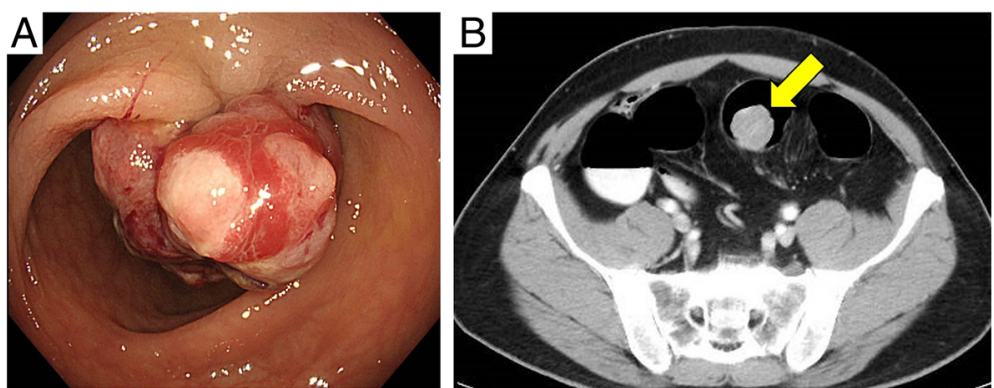

Fig. 1 a Endoscopic findings: colonoscopy revealed a large polypoidal tumor in the sigmoid colon. b Contrast-enhanced computed tomography findings: the position of the tumor is indicated; no metastasis to lymph nodes or a distant site was evident

examination including palpation was normal. The levels of carcinoembryonic antigen $(4.2 \mathrm{ng} / \mathrm{mL})$ and carbohydrate antigen 19-9 (13.2 U/mL) (commonly measured blood tumor markers) were normal. Colonoscopy revealed a polypoid lesion approximately $30 \mathrm{~mm}$ in diameter in the sigmoid colon $30 \mathrm{~cm}$ from the anal verge (Fig. 1a). Histopathological examination of a biopsy specimen revealed spindle-shaped cells exhibiting significant nuclear atypia and a trabecular proliferation pattern on hematoxylin-eosin staining. Immunohistochemically, the tissue was positive for SMA and desmin and negative for c-kit, DOG-1, CD34, and S-100. Furthermore, the Ki-67 index was $>50 \%$ (Fig. 2). Contrast-enhanced computed tomography from the chest to the pelvis revealed a tumor $28 \mathrm{~mm}$ in diameter in the sigmoid colon and the absence of involved lymph nodes and any distant metastasis (Fig. 1b).

We diagnosed an LMS of the sigmoid colon without metastasis. We performed laparoscopic sigmoid colectomy and regional lymphadenectomy, following the concept of complete mesocolic excision and high-level central vascular ligation with curative intent for colon cancer patients. Laparoscopic surgery was performed with the aid of five trocars. The first trocar $(12 \mathrm{~mm}$ in length) was placed in the umbilicus using an open method. Another 12-mm trocar was placed in the right lower abdomen, and three 5-mm trocars were placed in the left lower and either side of the abdomen (Fig. 3a). We identified the tattoo injected near the tumor before surgery (Fig. $3 \mathrm{~b}$ ). We dissected and mobilized the sigmoid colon by the medial-to-lateral approach in Toldt's space and performed high-level central vascular (inferior mesenteric artery) ligation (Fig. 3c). The sigmoid colon was transected to remove a $10-\mathrm{cm}$ tract margin from the tumor. After complete curative resection, a colorectal end-to-end anastomosis was created using the double-stapling technique (Fig. 3d). The tumor dimensions were $42 \times 37 \times 28$ $\mathrm{mm}$, and the surface was elastic and hard (Fig. 4a). The cross-section was white with a $5 \mathrm{~mm}$ peduncle (Fig. 4b). The epithelium was widely exfoliated, and the tumor per se featured trabecular proliferation of
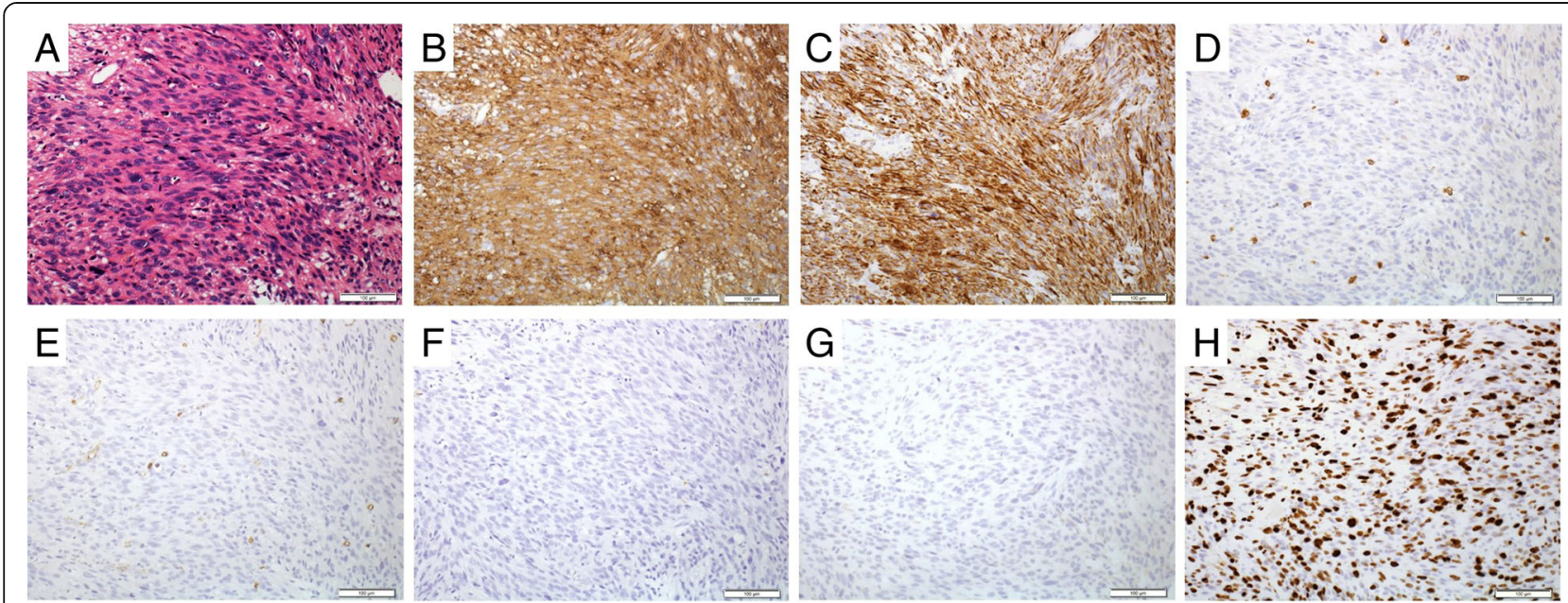

G

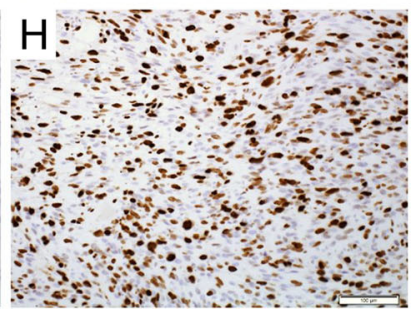

Fig. 2 Microscopic and immunohistochemical findings. a Hematoxylin-eosin staining revealed spindle-shaped cells exhibiting significant nuclear atypia. The tumor was immunohistochemically positive for a-SMA (b), desmin (c), and Ki-67 (h), but negative for c-kit (d), CD34 (e), S-100 (f), and DOG-1 (g) $(\times 20)$ 

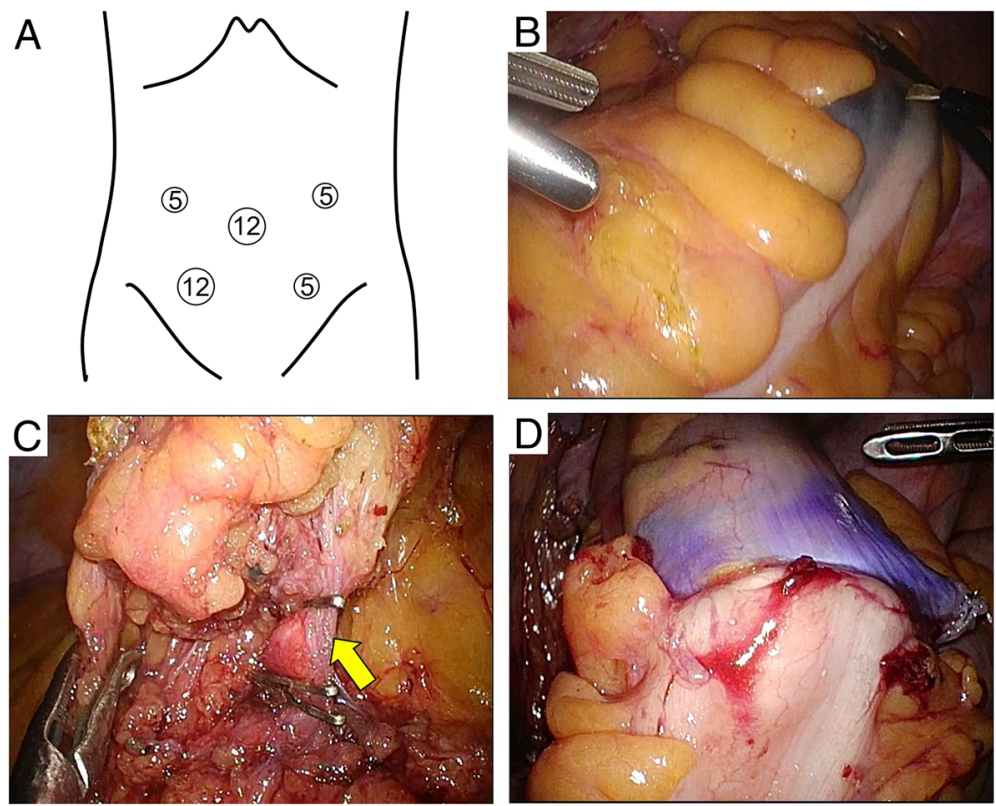

Fig. 3 The sites of the portals and intraoperative views. a Portal sites: the first trocar was a 12-mm umbilical trocar and another such trocar was placed in the right lower abdomen, followed by three $5-\mathrm{mm}$ trocars in the left lower and both sides of the abdomen. $\mathbf{b}$ The tattoo indicates the tumor site. c The arrow indicates the central ligation point of the inferior mesenteric artery. $\mathbf{d}$ Anastomosis was performed using the double-stapling technique
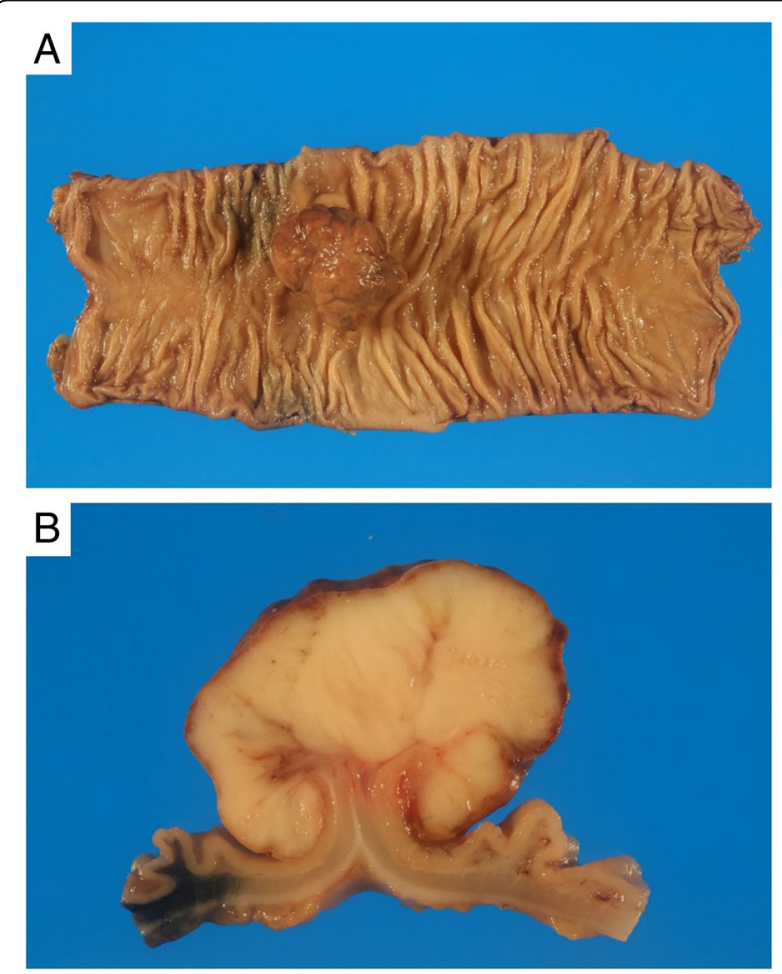

Fig. 4 The resected specimen. a The tumor dimensions were $42 \times 37 \times$ $28 \mathrm{~mm}$ and the surface was elastic and hard. $\mathbf{b}$ The cross-section was white with a 5 -mm peduncle spindle cells with prominent anisonucleosis and nuclear atypia. The tumor involved the base of the muscularis propria, but was not continuous with the base. Therefore, the tumor was considered to have originated in the muscularis mucosa. All surgical margins, and lymph node vascular invasion status, were negative. Forty-four lymph nodes were harvested; no lymph node metastasis was detected. The tumor was of histological grade 2 by reference to the French Federation Nationale des Centers de Lutte Contre le Cancer (FNCLCC) system [5]; of stage IIA of the TNM classification of the 7th Edition of the American Joint Committee on Cancer (AJCC) system [6], the 7th Edition of the Union for International Cancer Control (UICC) system, and a previously published surgical staging system [7].

The postoperative course was uneventful, and the patient was discharged 9 days after the operation. He was followed-up every 6 months by contrast-enhanced computed tomography and every 12 months by colonoscopy; he did not receive adjuvant chemotherapy. There was no

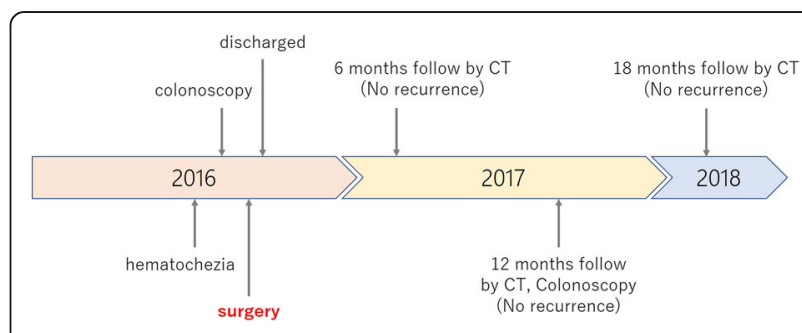

Fig. 5 Timeline of clinical course: CT computed tomography 


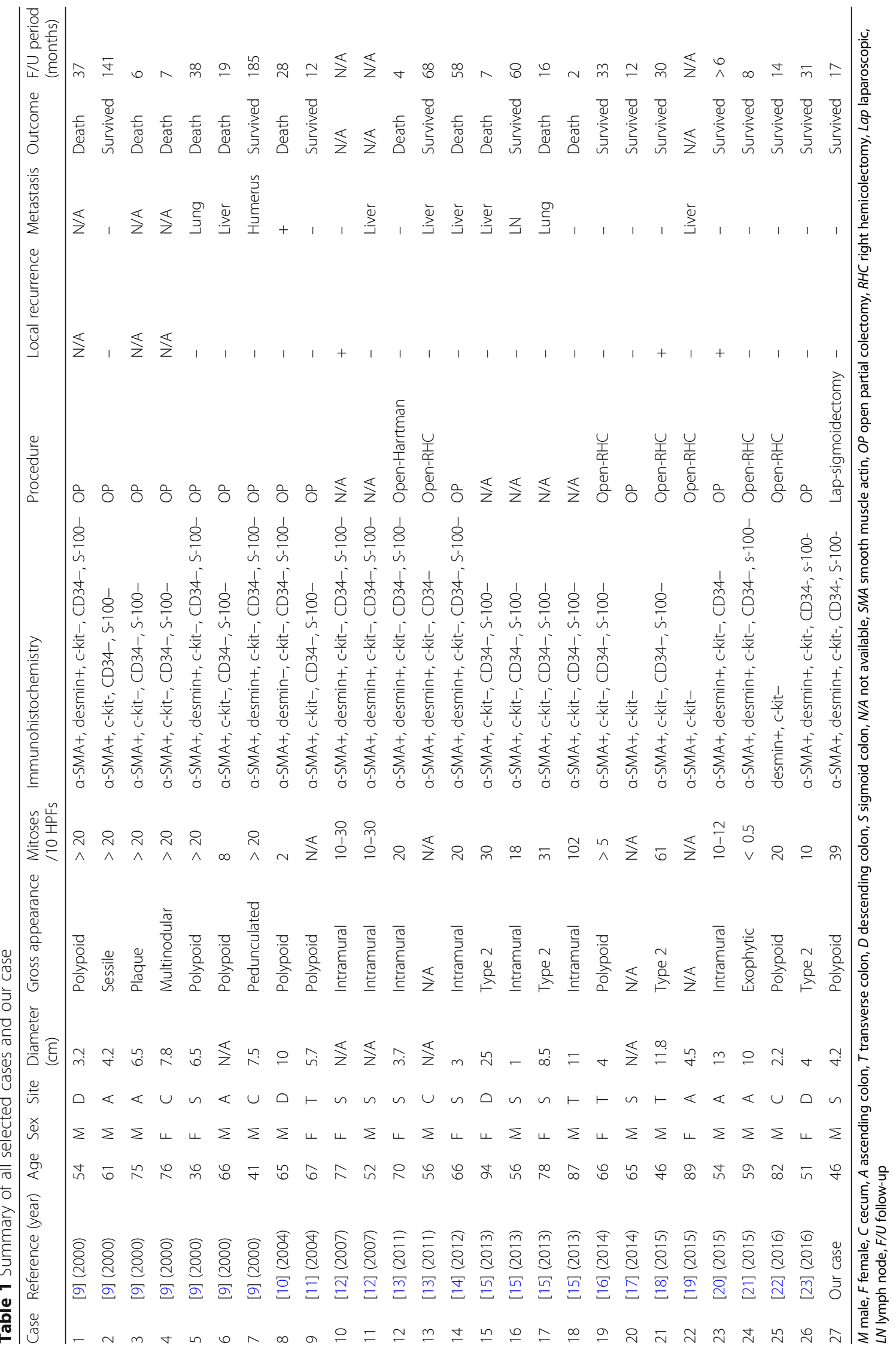


evidence of recurrence to 1.5 years after surgery. The clinical course of this patient is shown in Fig. 5.

LMS of the colon is often diagnosed later than LMS of the rectum. Therefore, only a few such patients can undergo laparoscopic surgery. We here report the first case of curative laparoscopic surgery to treat LMS of the colon.

LMSs originate from smooth muscle, which is widespread in the body; LMSs are most common in the retroperitoneum (including the pelvis), large blood vessels (principally the inferior vena cava), and the lower extremities [8]. Gastrointestinal LMSs of the colon are very uncommon, accounting for $<0.1 \%$ of all colorectal malignancies. Theoretically, LMSs can develop from both the muscularis propria and the muscularis mucosae; however, few reports on LMS origins have appeared. Although the muscularis propria contains more smooth muscle than the muscularis mucosae, a macroscopic polypoid LMS protruding inside the colon has often been reported [2]. Therefore, development of LMS from the muscularis mucosae (as in our case) may not be uncommon.

We searched the PubMed database using the MeSH terms "leiomyosarcoma" and "colon." English-language reports published after 1998 investigating cases that were clearly immunohistochemically diagnosed as LMSs were selected. All cited references were also reviewed. The search yielded 197 publications, of which 26 were ultimately evaluated [9-23]. Table 1 summarizes these data and our case. The patients included 16 males and 11 females of median age 57.5 years [interquartile range (IQR), 53-65.5 years]. Tumors were in the right colon in 14 cases, and the left colon in 13, most frequently in the sigmoid colon (9 cases). The median tumor diameter was $7 \mathrm{~cm}$ (IQR, $3.7-10.5 \mathrm{~cm}$ ).

Surgery is the standard treatment for localized soft tissue and visceral sarcomas [24]. In all cases, surgery was performed, but no standard therapeutic strategy for gastrointestinal LMSs has yet been established. Lymph node metastasis of gastrointestinal LMSs is rather uncommon; however, lymph node dissection is advisable if this is not excessively invasive, because lymph node metastasis has been reported even in those with small poorly proliferating tumors [one such tumor was $1 \mathrm{~cm}$ in diameter and exhibited 18 mitoses/50 high-power fields (HPFs)] [15]. The standard chemotherapies for advanced soft tissue and visceral sarcomas are first-line anthracyclines, with doxorubicin-plus-dacarbazine as an alternative [24]. Presently, chemotherapy plays a limited role in LMS treatment. Factors prognostic of LMS are unclear because LMSs are rare, but tumor diameter $\geq 5 \mathrm{~cm}$ has been reported to be poorly prognostic [15]. Therefore, minimally invasive laparoscopic surgery should be performed only when LMSs are $<5 \mathrm{~cm}$ in diameter, as in our case.

\section{Conclusion}

We report an LMS of the colon treated via laparoscopic surgery. Although further work is necessary to assess prognosis and to develop the treatment further, laparoscopic surgery to treat small colonic LMSs is feasible, minimally invasive, and curative.

\section{Abbreviations}

AJCC: American Joint Committee on Cancer; FNCLCC: French Federation Nationale des Centers de Lutte Contre le Cancer; GIST: Gastrointestinal stromal tumor; HPF: High-power field; IQR: Interquartile range; LMS: Leiomyosarcoma; SMA: Smooth muscle actin; UICC: Union for International Cancer Control

Acknowledgments

Not applicable.

\section{Funding}

There is no funding support for this manuscript.

Availability of data and materials

The datasets generated during the current study are not publicly available because individual privacy could be compromised, but are available from the corresponding author on reasonable request.

\section{Authors' contributions}

MY and YI wrote the manuscript. MW critically revised the manuscript. MY and $\mathrm{YI}$ performed the surgical treatment for this patient. AH contributed to the pathological examination. All authors have read and approved the manuscript.

Ethics approval and consent to participate

Kitasato University Kitasato Institute Hospital Ethics Committee approves and consents to publish this case report (No. 17008).

\section{Consent for publication}

Written consent to publish was obtained from the patient of this case report.

\section{Competing interests}

The authors declare that they have no competing interests.

\section{Publisher's Note}

Springer Nature remains neutral with regard to jurisdictional claims in published maps and institutional affiliations.

\section{Author details}

${ }^{1}$ Department of Surgery, Kitasato University Kitasato Institute Hospital, 5-9-1 Shirokane, Minato-ku, Tokyo, Japan. ${ }^{2}$ Department of Pathology, Kitasato University Kitasato Institute Hospital, 5-9-1 Shirokane, Minato-ku, Tokyo, Japan. ${ }^{3}$ Department of Surgery, Kitasato University School of Medicine, 1-15-1 Kitasato, Sagamihara, Kanagawa, Japan.

Received: 22 October 2018 Accepted: 31 January 2019

Published online: 12 February 2019

References

1. Walsh TH, Mann CV. Smooth muscle neoplasms of the rectum and anal canal. Br J Surg. 1984;71(8):597-9.

2. Aggarwal G, Sharma S, Zheng M, Reid MD, Crosby JH, Chamberlain SM, et al. Primary leiomyosarcomas of the gastrointestinal tract in the postgastrointestinal stromal tumor era. Ann Diagn Pathol. 2012;16(6):532-40.

3. Hirota S, Isozaki K, Moriyama Y, Hashimoto K, Nishida T, Ishiguro S, et al. Gain-of-function mutations of c-kit in human gastrointestinal stromal tumors. Science. 1998;279(5350):577-80.

4. Miettinen M, Sarlomo-Rikala M, Lasota J. Gastrointestinal stromal tumors: recent advances in understanding of their biology. Hum Pathol. 1999;30(10):1213-20.

5. Trojani M, Contesso G, Coindre JM, Rouesse J, Bui NB, de Mascarel A, et al. Soft-tissue sarcomas of adults; study of pathological prognostic variables and definition of a histopathological grading system. Int J Cancer. 1984;33(1):37-42. 
6. Edge SB, Compton CC. The American Joint Committee on Cancer: the 7th edition of the AJCC cancer staging manual and the future of TNM. Ann Surg Oncol. 2010;17(6):1471-4.

7. Enneking WF, Spanier SS, Goodman MA. A system for the surgical staging of musculoskeletal sarcoma. Clin Orthop Relat Res. 1980;2003(415):4-18.

8. Duffaud F, Ray-Coquard I, Salas S, Pautier P. Recent advances in understanding and managing leiomyosarcomas. F1000Prime Rep. 2015;7:55.

9. Miettinen M, Sarlomo-Rikala M, Sobin LH, Lasota J. Gastrointestinal stromal tumors and leiomyosarcomas in the colon: a clinicopathologic, immunohistochemical, and molecular genetic study of 44 cases. Am J Surg Pathol. 2000;24(10):1339-52.

10. Insabato L, Di Vizio D, Ciancia G, Pettinato G, Tornillo L, Terracciano L. Malignant gastrointestinal leiomyosarcoma and gastrointestinal stromal tumor with prominent osteoclast-like giant cells. Arch Pathol Lab Med. 2004;128(4):440-3.

11. Michalopoulos A, Papadopoulos VN, Basdanis G, Haralabopoulos E, Zatagias A, Netta $S$, et al. Colorectal gastrointestinal mesenchymal tumours. Report of a stromal case of the rectum (GIST) and a leiomyosarcoma of the transverse colon. Tech Coloproctol. 2004;8(Suppl 1):s155-7.

12. Agaimy A, Wunsch PH. True smooth muscle neoplasms of the gastrointestinal tract: morphological spectrum and classification in a series of 85 cases from a single institute. Langenbeck's Arch Surg. 2007;392(1):75-81.

13. Resch T, Oberhuber R, Zitt M, Laimer E, Gehwolf P, Pratschke J, et al. Leiomyosarcoma of the colon: unresolved issues of a rare but highly aggressive malignancy. Am Surg. 2011;77(4):E62-4.

14. Hamai Y, Hihara J, Emi M, Aoki Y, Kushitani K, Tanabe K, et al. Leiomyosarcoma of the sigmoid colon with multiple liver metastases and gastric cancer: a case report. BMC Gastroenterol. 2012;12:98.

15. Yamamoto H, Handa M, Tobo T, Setsu N, Fujita K, Oshiro Y, et al. Clinicopathological features of primary leiomyosarcoma of the gastrointestinal tract following recognition of gastrointestinal stromal tumours. Histopathology. 2013;63(2):194-207.

16. Yaren A, Degirmencioglu S, Calli Demirkan N, Gokcen Demiray A, Taskoylu B, Dogu GG. Primary mesenchymal tumors of the colon: a report of three cases. Turk J Gastroenterol. 2014;25(3):314-8.

17. Abdel Samie A, Sun R, Fayyazi A, Theilmann L. Leiomyosarcoma of the sigmoid colon: a rare cause of intestinal intussusception. J Gastrointest Cancer. 2014;45(Suppl 1):6-9.

18. Kono M, Tsuji N, Ozaki N, Matsumoto N, Takaba T, Okumura N, et al. Primary leiomyosarcoma of the colon. Clin J Gastroenterol. 2015;8(4):217-22.

19. Granero-Peiro L, Martinez-Ortega P, Sanchez-Justicia C, Hernandez-Lizoain $J$ L. Leiomyosarcoma of the ascending colon: a rare tumor with poor prognosis. Rev Esp Enferm Dig. 2015;107(9):584-5.

20. Kiran P, Shiny PM, Dhanya KS, Aravindan KP. Diagnosis of leiomyosarcoma of colon. J Cancer Res Ther. 2015;11(4):1035.

21. Janevski V, Selmani R, Janevska V, Spasevska L, Zhivadinovik J. Leiomyosarcoma of the colon. Med Pregl. 2015;68(11-12):413-7.

22. Kim VM, Goicochea L, Fang SH. Case report: collision tumour of colon leiomyosarcoma and adenocarcinoma. J Clin Diagn Res. 2016;10(6):Pd03-4.

23. Akutsu D, Mizokami Y, Suzuki H, Terasaki M, Narasaka T, Kaneko T, et al. A rare case of colonic leiomyosarcoma in association with ulcerative colitis. Intern Med. 2016;55(19):2799-803.

24. ESMO/European Sarcoma Network Working Group. Soft tissue and visceral sarcomas: ESMO Clinical Practice Guidelines for diagnosis, treatment and follow-up. Ann Oncol. 2014;25(Suppl 3):iii102-12.

\section{Submit your manuscript to a SpringerOpen ${ }^{\circ}$ journal and benefit from:}

- Convenient online submission

- Rigorous peer review

- Open access: articles freely available online

- High visibility within the field

- Retaining the copyright to your article

Submit your next manuscript at $\boldsymbol{\nabla}$ springeropen.com 